\title{
Artificial Science
}

Elton N. Kaufmann

Modern scientific research demands modern laboratory instrumentation and associated know-how. Ever increasing levels of sophistication are required of our instruments... we need atomic resolution microscopy, more intense and broader-spectral photon sources, colder neutrons, etc. There will always be a place in the lab for the likes of the basic volt-ohmmeter, the calorimeter and the oscilloscope, adorned or not by the many new "bells and whistles" modern microelectronics can provide. But the frontiers of knowledge in many fields, including materials research, have clearly progressed to where further basic understanding awaits experiments only possible with the very newest, most powerful and very expensive methods.

The expense, of course, is what necessitates prolonged debate over priorities and lengthy and repeated justification of major equipment (and associated construction) proposals. An institution commits resources, personnel, and prestige to such acquisitions and, once acquired, must continue to aggressively seek sustaining operational support. I think most would agree that science and technology have realized substantial net benefit from those state-of-the-art resources already in existence and probably will enjoy analogous benefits from capabilities still in proposal or drawing-board stages.

This is true whether or not (by the time new facilities come on-line years after conception) the original justifying research purpose is still the hot topic and initial mission. There is the usually unspoken general recognition that it is, in the end, the facility/capability which is the real goal and, if the justifying means to the end are forgotten along the way, there will be equally or more exciting and important science to tackle with the new machines when they're ready. That is, an instrumental capability is, in a very real sense, generic. It is, by definition, good, just as the voltohmmeter is good, and similarly, comes to be regarded as indispensable once it exists.

When an exciting, important scientific or technological question does not demand the application of the new expensive, well-staffed facility, the facility demands or invents the question. Not only must continued financial support be assured, but with any facility is associated a following - i.e., scientists whose specialty and primary interests are in expert application of "the technique," whatever it happens to be. These experts play a crucial role in the efficient and productive use of a facility, but they are not universally portable. Thus, not only in slack times, but all the time, such experts think first, "How can we apply our method to this problem?" rather than, "Does this problem need our method?"

\section{Rather than viewing technique-generated research as self-justifying "make-work" to fill gaps between truly useful programs, we might recognize it as an opportunity.}

The best current example of the phenomena is in high $T_{c}$ superconductors. $\mathrm{Y}$-Ba-Cu-oxide has probably been subjected to every analysis method and prepared by every synthesis technique known. Why? Because, in some cases, logic based on past experience with ceramics and/or superconductors dictated the approach. In other cases a "what if we try using our method on this stuff" syndrome seems to have operated.

After generations of techniquedictated problems, it's easy to forget the unnatural origins of some of our science ... take radiation effects and atomic collisions in solids, for example. These pursuits were not launched as fields by natural radium ores and cosmic rays but by the advent of particle accelerators and nuclear reactors. Technologyrelevant problems created the interest in fundamental mechanisms and, as a byproduct, many "technique-created phenomena" have been studied as scientific curiosities as well.

So what began as part of natural philosophy has, through advances in instrumental capability, been multiply folded back on itself to where the targets of research are decidedly unnatural. Some paths may lead only to short-lived projects and obscure publications but many lead adventitiously or through educated intuition to artificially structured systems which form the core of modern technology. Thus, rather than viewing technique-generated research as self-justifying "make-work" to fill gaps between truly useful programs, we might recognize it as an opportunity. Scientists yearn for the times when they had freedom to do the what-if experiments and follow their noses. The imperative of full facility utilization* now demands the what-if experiment and may therefore be the new freedom allowing discovery and innovation.

* Of course, the largest and newest facilities are few in number and over subscribed with long waiting lists of proposals. They are nevertheless liable to experience slumps in demand later in their useful lives and be prodigious sources of artificial science.

Elton N. Kaufmann is associate division leader, Materials Division, Lawrence Livermore National Laboratory, and chairman of the MRS BULLETIN Editorial Board.

\section{Send your comments and views on issues affecting materials research to: \\ Editor, MRS BULLETIN \\ 9800 McKnight Road, Suite 327 \\ Pittsburgh, PA 15237 \\ Telephone (412) 367-3036 \\ Fax (412) 367-4373}

